\title{
Symmetry Allows for Distinguishability in Totally Destructive Many-Particle Interference
}

\author{
Julian Münzberg $\odot,{ }^{1, *}$ Christoph Dittel $\odot,{ }^{2,3}$ Maxime Lebugle, ${ }^{4}$ Andreas Buchleitner, ${ }^{2,3}$ \\ Alexander Szameit $\odot,{ }^{5}$ Gregor Weihs $\odot,{ }^{1}$ and Robert Keil ${ }^{1}$ \\ ${ }^{1}$ Institut für Experimentalphysik, Universität Innsbruck, Technikerstr. 25, Innsbruck 6020, Austria \\ ${ }^{2}$ Physikalisches Institut, Albert-Ludwigs-Universität Freiburg, Hermann-Herder-Str. 3, Freiburg 79104, Germany \\ ${ }^{3}$ EUCOR Centre for Quantum Science and Quantum Computing, Albert-Ludwigs-Universität Freiburg, \\ Hermann-Herder-Str. 3, Freiburg 79104, Germany \\ ${ }^{4}$ Eulitha, Studacherstrasse 7b, Kirchdorf 5416, Switzerland \\ ${ }^{5}$ Institut für Physik, Universität Rostock, Albert-Einstein-Str. 23, Rostock 18059, Germany
}

(Received 22 February 2021; accepted 21 April 2021; published 25 May 2021)

\begin{abstract}
We investigate, in a four-photon interference experiment in a laser-written waveguide structure, how symmetries control the suppression of many-body output events of a $J_{x}$ unitary. We show that totally destructive interference does not require mutual indistinguishability between all but only between symmetrically paired particles, in agreement with recent theoretical predictions. The outcome of the experiment is well described by a quantitative simulation that accounts for higher-order emission of the photon source, imbalances in the scattering network, partial distinguishability, and photon loss.
\end{abstract}

DOI: 10.1103/PRXQuantum.2.020326

\section{INTRODUCTION}

Many-particle interference lies at the very heart of many quantum information and computations schemes with photons [1-3], since coherent superpositions of many-particle states can accommodate a level of complexity that is out of reach for deterministic classical simulations [4-16]. A necessary condition for quantum interference on the manyparticle level is the indistinguishability of distinct manyparticle transition amplitudes, thus lifting wave-particle duality [17] from the single particle to the many-particle level [18].

Importantly, such indistinguishability of amplitudes relies on two distinct aspects of the specific physical setting - the topologies of the superimposed amplitudes and the indistinguishabilities of the involved particles. The topology, determined by the external potential seen by the particles, determines the actual number of particles that potentially populate the contributing many-particle transition amplitudes and thereby defines the subsets of particles that ought to be mutually indistinguishable for

*julian.muenzberg@uibk.ac.at

Published by the American Physical Society under the terms of the Creative Commons Attribution 4.0 International license. Further distribution of this work must maintain attribution to the author(s) and the published article's title, journal citation, and DOI. many-particle interference to impact on the experimentally accessible counting statistics.

In the paradigmatic setting of two-particle interference due to Hong, Ou, and Mandel (HOM) [19], where two photons, with a controllable degree of (in)distinguishability, each enter one of the input modes of a balanced beam splitter, the topological aspect remains trivial, since only two two-particle transition amplitudes (both photons reflected or both photons transmitted at one single symmetric potential barrier) are coupled and the experimental phenomenology thus hinges entirely on the indistinguishability of the incoming photons. Consequently, the famous strict suppression of the coincident output event, with one particle detected in each output port, reliably certifies that indistinguishability.

A generalization of the HOM setting to larger numbers of modes and particles is possible, under suitable symmetry requirements for the (single-particle) unitary that defines the noninteracting many-particle scattering process. In such a scenario so-called suppression laws [12,2024] identify those transition events that are strictly suppressed by destructive many-particle interference. These have been validated experimentally [25-32] and a theoretical formulation $[33,34]$ has been found that - by algebraic considerations and hence applicable for arbitrary system sizes - anchors them to the symmetry properties of the many-particle input state and the scattering unitary under permutations. Since symmetry considerations reduce the complexity of the general many-particle 
interference problem, the experimental validation of said suppression rules has been suggested as a viable certification protocol for bona fide many-particle interference phenomena, as it directly assesses the granular features of many-particle quantum interference, in contrast, e.g., to mean-field samplers [27,30,35-37].

However, because of the, in general, nontrivial imprint of the topology of the transition amplitudes, it is important to realize that many-body interference effects do not necessarily require mutual indistinguishability of all involved particles [38]. Specifically, for the many-particle suppression rules, this implies that many-particle transmission events can be perfectly suppressed even for pairwise perfectly distinguishable subsets of particles, if only the topology of the superimposed transition amplitudes remains invariant under their exchange, which is ultimately controlled by the symmetry properties of the injected manyparticle state [39].

Here, we experimentally unfold this refinement of the physics underlying the many-particle suppression laws, by implementing the evolution of four photons under the so-called $J_{x}$ unitary transformation, for different mutual distinguishabilities between the particles. So far, this scattering scenario has only been investigated experimentally with photon pairs, making it impossible to distinguish between the effect of symmetry and full indistinguishability $[22,29]$. However, by using four photons, we show here that the suppression effect persists as long as the initial state exhibits the required symmetry, even if two pairs of indistinguishable particles are made fully distinguishable. Our findings are in agreement with recent theoretical insights and open up new perspectives in the characterization and validation of many-body indistinguishability.

This work is structured as follows. In Sec. II, we recap the suppression law for the $J_{x}$ unitary and introduce the experimentally investigated input states differing due to the indistinguishability of the particles. Depending on these states, the scenario is expected to show either suppression or no suppression. Section III describes the experimental setup and outlines the measurement procedure. We present the experimental results in Sec. IV and conclude in Sec. V. Details on further aspects of the measurement procedure and the theoretical model describing our experiment are deferred to the Appendix.

\section{SUPPRESSION LAW FOR THE $\boldsymbol{J}_{x}$ UNITARY}

Let us consider a set of $N$ bosons initially prepared in a multimode Fock state with respect to the spatial input modes of an optical scattering device. The latter implements a unitary transformation that redistributes the particles over its $n$ modes, with the many-particle output state analyzed by projective measurements of the occupation numbers of the individual modes. Given that the unitary is invariant under mode permutations and that the input state satisfies a related permutation-symmetry, the general suppression laws formulated in Refs. [33,34] specify which input-output combinations are suppressed by totally destructive many-particle interference.

The so-called $J_{x}$ unitary satisfies such permutation symmetry and, according to the general suppression laws, exhibits a large number of suppressed output events for input states that are mirror symmetric with respect to the central mode. While a detailed derivation of the $J_{x}$ suppression law can be found in Refs. [34,39], in the following we summarize the main ingredients that are relevant for our work.

The single-particle $J_{x}$ unitary $U^{J}(t)=e^{i J_{x} t / \hbar}$ is generated by the angular-momentum operator $J_{x}$ in the $x$ direction, with matrix elements $[22,29,40]$

$$
\left[J_{x}\right]_{k, j}=\frac{\hbar}{2}\left(\sqrt{k(n-k)} \delta_{j, k+1}+\sqrt{j(n-j)} \delta_{j, k-1}\right),
$$

where $\delta_{j, k}$ is the Kronecker delta. For an evolution time $t=$ $\pi / 2$, the resulting unitary $U^{J}(\pi / 2) \equiv U^{J}$ appears, up to a phase factor, mirror symmetric with respect to the central mode $[34,39]$ :

$$
U_{k, \pi^{J}(j)}^{J}=U_{k, j}^{J} \exp \left(i \pi\left[k-j+\frac{n-1}{2}\right]\right),
$$

in which

$$
\pi^{J}(j)=n+1-j
$$

is the mirror-symmetric permutation of modes $j \in$ $\{1, \ldots, n\}$ and $\mathscr{P}^{J}$ the corresponding single-particle permutation operator [41]. Given that the unitary evolution and the measurement of the output-mode occupation of the particles only act upon their external degrees of freedom, i.e., their mode indices, we can trace out all remaining - internal - degrees of freedom that, potentially, render them partially distinguishable. Note that in our photonic setting, the internal degrees of freedom include, for example, the polarization, spectral properties, and arrival times of the photons. This procedure results in the reduced external $N$-particle density operator $\rho_{E}$ (for details, see Refs. [18,39] and Appendix A). For all mirror-symmetric input states with uncorrelated internal degrees of freedom (see Appendices A.1 and A.3) and an external state $\rho_{E}$ satisfying

$$
\left[\left(\mathscr{P}^{J}\right)^{\otimes N}, \rho_{E}\right]=0,
$$

we retrieve the following suppression law: All output states with an odd number of particles in even output modes are suppressed [34].

Note that Eq. (4) does not necessarily require the mutual indistinguishability of all constituent particles. In 
the case of internal product states, only particles occupying modes that belong to the same cycle $c$ of $\pi^{J}$-and thus define those subsets of particles that mutually interfere to induce the suppression of the output events predicted by (4) - must share the same internal state for (4) to hold [42].

In our experiment, we demonstrate the relation between the symmetry of the input state [Eq. (4)] and the suppression of many-body output events for the interference of $N=4$ photons on a $J_{x}$ unitary with $n=7$ modes. In cycle notation, the mode permutation (3) then reads $\pi^{J}=$ (17)(26)(3 5)(4), with cycles $c_{1}=(17), c_{2}=(26), c_{3}=$ (35), and $c_{4}=(4)$. We investigate four different cases of the indistinguishability structure of the particles, as illustrated in Fig. 1(a). These scenarios correspond to the particles on input being mutually indistinguishable, intercycle distinguishable, intracycle distinguishable, and mutually distinguishable. Even though the second scenario features distinguishable particles in modes belonging to distinct

(a)

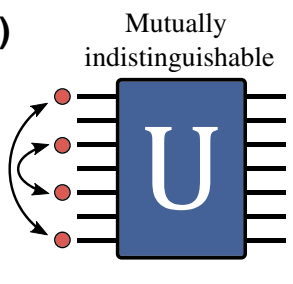

Intracycle distinguishable

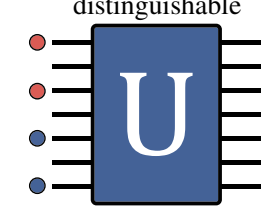

(b)



(c)

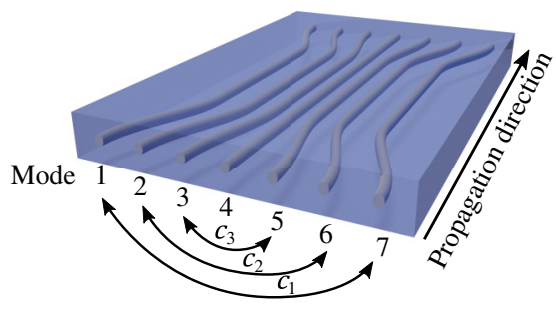

FIG. 1. The experimentally investigated input states of the $J_{x}$ unitary. (a) Photons are input to the odd modes of the unitary. Four different symmetry scenarios with respect to the mirror axis (central mode) are illustrated. Suppression is expected only for the upper two scenarios. The internal states of the particles are illustrated by their coloring. (b) The occupation of the permutation cycles $c_{j}$. (c) The photonic waveguide structure implementing the $J_{x}$ unitary. cycles, it remains invariant under $\pi^{J}$ and, thus, gives rise to the above suppression law [compare Fig. 1(b)]. In contrast, the latter two input conformations cannot give rise to the suppression effect under scrutiny here. The counting statistics observed in our experimental analysis unambiguously reveal the symmetry-induced minimal requirements on the mutual indistinguishability only of subsets of the incoming $\mathrm{N}$-particle state for interference-induced output event suppression.

\section{EXPERIMENTAL SETUP}

The many-particle interference experiment is performed on an integrated linear-optics platform. For the implementation of the $J_{x}$ unitary, we use an array of seven evanescently coupled modes in a fs laser-written waveguide structure in fused silica $[43,44]$. The waveguide chip has a total length of $150 \mathrm{~mm}$, of which $61.4 \mathrm{~mm}$ is occupied by the central interaction region, while the fanin and fan-out sections are $39.6 \mathrm{~mm}$ and $49 \mathrm{~mm}$ long, respectively. The evanescent coupling between the waveguides is chosen according to the nearest-neighbor coupling structure from Eq. (1), with the 61.4-mm interaction length corresponding to an evolution time $t=\pi / 2$. The desired coupling strengths are produced by varying the distance between adjacent waveguides within the coupling region (mode field diameters about $18 \mu \mathrm{m} \times 20 \mu \mathrm{m}$ ) from approximately $35.5 \mu \mathrm{m}$ for the outermost waveguides to $32 \mu \mathrm{m}$ for the innermost waveguides. A schematic representation of the structure is shown in Fig. 1(c). For details on the waveguide fabrication, see Appendix B.

The four-photon input state is generated by doublepair emission of a type-I spontaneous parametric downconversion (SPDC) source [45]. The experimental setup is illustrated in Fig. 2. A 200-fs-pulsed Ti:Sa laser with a repetition rate of $76 \mathrm{MHz}$ and a center wavelength of 795 $\mathrm{nm}$ is frequency doubled in a bismuth borate (BiBO) crystal. The resulting up-converted $V$-polarized light with an optical power of approximately $150 \mathrm{~mW}$ is focused into a beta barium borate (BBO) crystal, where photon pairs are created in a type-I noncollinear SPDC process. The emitted $H$-polarized photon pairs at a center wavelength of 795 $\mathrm{nm}$ are collected into four polarization-maintaining singlemode fibers after spectral filtering using 3-nm FWHM band-pass filters, which reduces spectral correlations and improves the indistinguishability between photons from different pairs. The four fibers, labeled as channels $A-D$, are oriented such that the channel pairs $A$ and $B$, as well as $C$ and $D$ (also called mode pairs), collect photons from opposite spots of the SPDC emission cone. Hence, by conservation of momentum, each mode pair collects both photons originating from the same SPDC event. The collection of two photon pairs within their coherence time (i.e., temporally overlapping wave packets) can arise from two different types of events: either the photon pairs are 


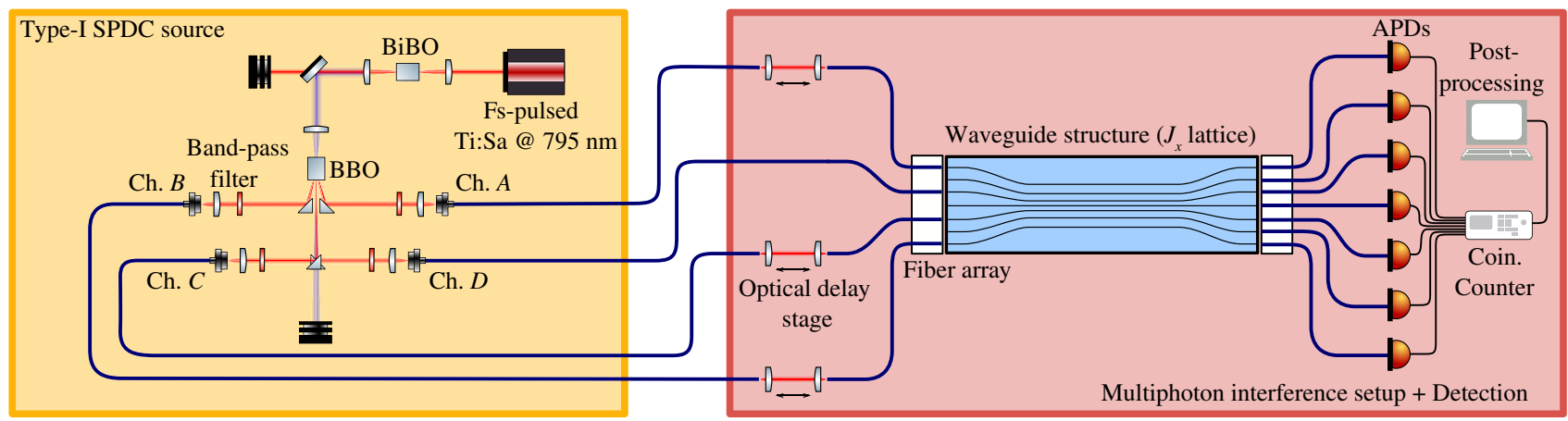

FIG. 2. A schematic illustration of the four-photon SPDC source, of the multiphoton interference, and of the detection setup. A pulsed Ti:Sa laser is frequency doubled by second-harmonic generation in a BiBO crystal in collinear configuration. The residual pump light is filtered by dichroic mirrors, while the up-converted light is focused into a BBO crystal, where pairs of photons are generated in a type-I SPDC process. The emitted and spectrally filtered photon pairs are collected via four polarization-maintaining single-mode fibers, labeled as channels (Ch.) $A-D$. Three free-space optical delay stages are used to adjust the temporal delay between photons from different channels. The light is coupled to the waveguide chip via a fiber array. The output of the waveguide structure is collected with multimode fibers and measured using APDs and a coincidence counter (Coin. Counter).

collected by the same mode pair, e.g., both channels $A$ and $B$ collect two photons, or by different mode pairs, such that each of channels $A-D$ collects one photon.

In the experiment, we connect channels $A$ and $B$ to input modes 1 and 7 of the $J_{x}$ unitary (i.e., to the modes of cycle $c_{1}$ ) and channels $C$ and $D$ to modes 3 and 5 (of cycle $c_{3}$ ), respectively. This connectivity is used for all four investigated scenarios. Hence, the photon-collection procedure results in the input-mode occupations

$$
\begin{aligned}
& \vec{R}_{1}=(2,0,0,0,0,0,2), \\
& \vec{R}_{2}=(1,0,1,0,1,0,1), \\
& \vec{R}_{3}=(0,0,2,0,2,0,0),
\end{aligned}
$$

where $\vec{R}_{j}$ lists the number of photons in each input mode. Note that in the ideal case of perfect indistinguishability between all particles, the states corresponding to all three possible input configurations satisfy the required mirror symmetry [see Eq. (3)]. In the experiment, however, there are residual spectral correlations, rendering photons from different pairs partially distinguishable [46]. Considering this, the described connectivity also ensures that photons from the same SPDC event always occupy modes belonging to the same cycle, such that the symmetry conditions are best satisfied in the presence of experimental imperfections.

To control the temporal delays between the photons and, thus, their mutual distinguishability, we use freespace optical delay stages in three of the four channels. The four scenarios shown in Fig. 1(a) are implemented by appropriately adjusting the time delays of the photons. For coupling light into the quasi-transverse-magnetic mode of the waveguide structure and collecting the outcome, we use a commercially available polarization-maintaining and multimode V-groove fiber array, respectively. The fibers of the output array are connected to seven avalanche photodiode (APDs), with an average detection efficiency of $65 \%$.

After having optimized the temporal overlap of photons from different channels in HOM-type measurements (for details, see Appendix C), we record all fourfold coincidence events with a time-tagging device. Only nonbunching output events (at most, one photon ends up in an output mode) are experimentally accessible, since the APDs used are non-number-resolving. In parallel to fourfold coincidences, we collect single-channel count rates, as well as twofold and threefold coincidences between all output modes (see Appendices D and E).

\section{RESULTS}

For each scenario shown in Fig. 1(a), we record fourfold coincidences between distinct output modes. Dependent on the scenario, the average event rate ranges between 0.0023 and $0.0045 \mathrm{~Hz}$, with a total number of collected events, $N_{\text {total }}$, between 566 and 1154 (see Table I). The experimental results are shown in Fig. 3, together with the theoretical predictions obtained from a simulation of the experiment. The experimental and simulated results are both normalized. In Figs. 3(a) and 3(b), all output events in the gray shaded area are suppressed in the ideal case, as predicted by the suppression law. While our data are substantially biased by higher-order multi-photon-pair emission (gray bars in Fig. 3; approximately 34\% of all registered fourphoton events can be attributed to the six-photon background), we can confirm a clear reduction of all ideally suppressed events in the case of mutually indistinguishable and intercycle distinguishable particles [see Figs. 3(a) and 3(b)], to be contrasted with the cases of intracycle and 
TABLE I. For the four investigated scenarios illustrated in Fig. 1(a), the measured $\left(\mathcal{D}_{\text {exp }}\right)$ and simulated $\left(\mathcal{D}_{\text {th }}\right)$ degree of suppression violation is listed, together with the total number $N_{\text {total }}$ of recorded four-photon coincidences.

\begin{tabular}{llll}
\hline \hline & $N_{\text {total }}$ & $\mathcal{D}_{\text {exp }}$ & $\mathcal{D}_{\text {th }}$ \\
\hline Mutually indistinguishable & 862 & $0.300 \pm 0.019$ & 0.214 \\
Intercycle distinguishable & 566 & $0.274 \pm 0.022$ & 0.226 \\
Intracycle distinguishable & 921 & $0.408 \pm 0.021$ & 0.414 \\
Mutually distinguishable & 1154 & $0.403 \pm 0.019$ & 0.415 \\
\hline \hline
\end{tabular}

mutually distinguishable particles [see Figs. 3(c) and 3(d)], where no suppression effect is expected or observed.

In order to benchmark the suppression, we use the degree of suppression violation $\mathcal{D}=N_{\text {forbidden }} / N_{\text {total }}$ [30], where $N_{\text {forbidden }}$ is the number of recorded four-photon coincidences corresponding to ideally suppressed output events and $N_{\text {total }}$ the total number of recorded events. Note that in the ideal case, $\mathcal{D}=0$. However, we expect a nonvanishing value of $\mathcal{D}$ due to fabrication errors of the waveguide structure, imperfections in the indistinguishability of the photons (caused by their spectrotemporal properties), and higher-order multipair emission.

In our theoretical simulation of the experiment, we account for the above experimental imperfections.
Pertinent to photon pairs from SPDC are their spectral correlations, arising from energy and conservation of momentum in the generation process, such that the combined internal state of all particles does not factorize into individual internal states of the particles [46]. Not only does this spectral entanglement influence the ratio between spontaneous and stimulated emission in the SPDC process and, thereby, the photon-number distribution [47-49], but it also strongly affects the many-particle interference for configurations with more than one photon occupying the same initial mode. The established method of calculation using the so-called partial indistinguishability matrix [50] is applicable to this situation but computationally costly, as it requires a double summation over all permutations of particle orderings. The more efficient formalism from Ref. [51], however, cannot be used here as it relies on factorizable internal states. Adopting the formalism of Ref. [39], here we derive a new framework for many-particle interference of correlated photon pairs, which avoids the costly summation over all particle orderings and replaces it by a faster summation over inequivalent orderings only. This framework is the first, to our knowledge, that simultaneously incorporates correlated internal states and multiple occupancy of external input modes, as well as the correct weights between stimulated and spontaneous emission in
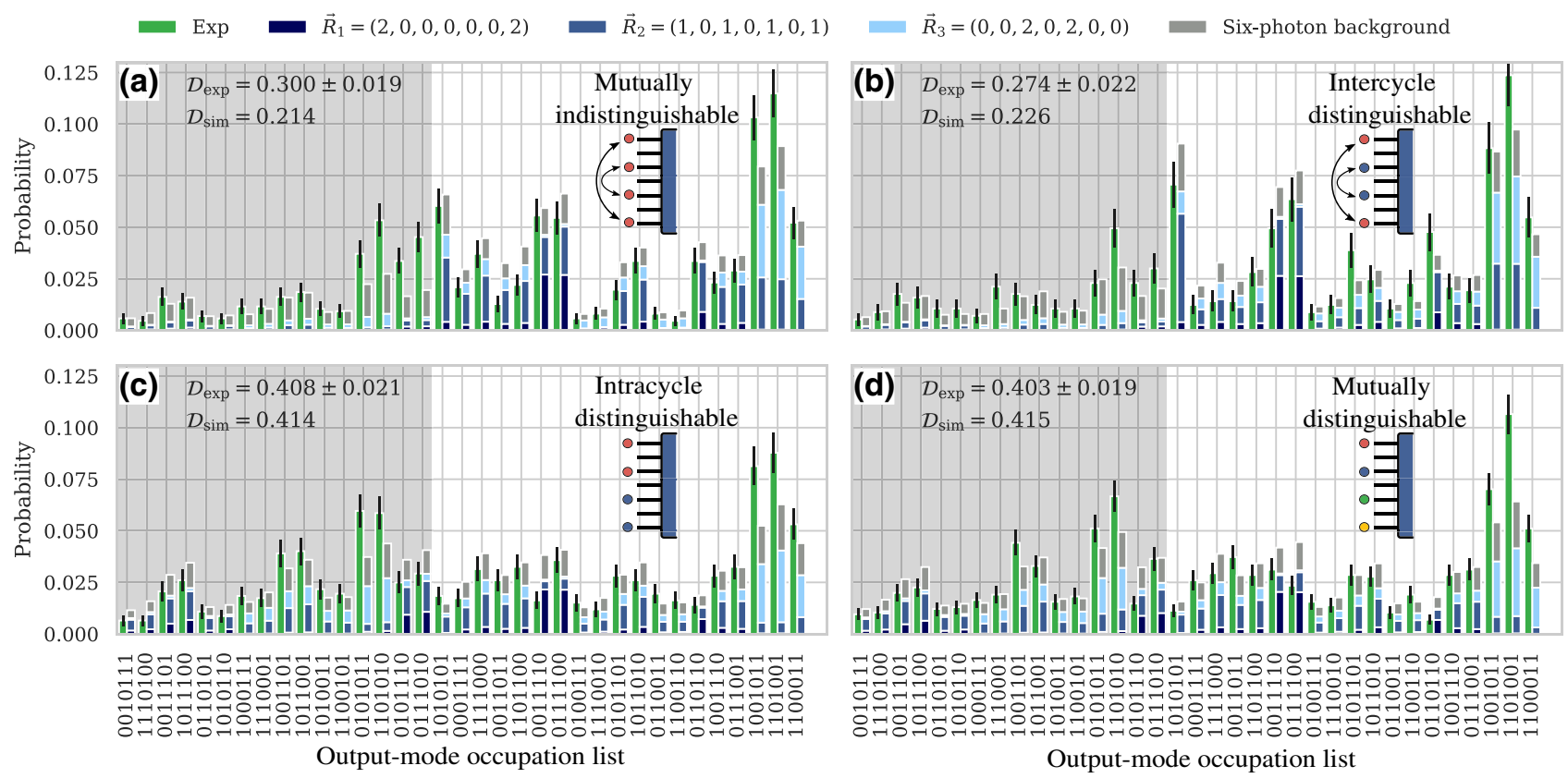

FIG. 3. The experimental and simulated output statistics of all fourfold coincidences between distinct output modes. (a),(b) The output statistics obtained for a mutually indistinguishable and for an intercycle distinguishable input state, respectively, for which all output events in the gray shaded area are ideally suppressed. (c),(d) The output statistics obtained for an intracycle distinguishable and for a mutually distinguishable input state, respectively. Here, no suppression effect is expected, even in the ideal case. The green bars correspond to experimental (Exp) data and the error bars indicate one standard deviation of the Poissonian counting statistics. The bars to the right of the experimental data correspond to a simulation of the experiment, with the dark blue, blue, light blue, and gray parts of the bars indicating the contribution stemming from the input-mode occupation $\vec{R}_{1}, \vec{R}_{2}, \vec{R}_{3}$ [see Eq. (5)] and from the six-photon background of the photon source, respectively. 
the various multipair contributions of the SPDC and is described in detail in Appendix A.

The experimentally measured and theoretically predicted degrees of suppression violation are summarized in Table I.

As quantified by the degree of suppression violation for both mutually indistinguishable and intercycle distinguishable particles, there is a significantly smaller fraction of events in the gray area of Fig. 3 compared to the cases of mutually distinguishable and intracycle distinguishable particles, which violate the mirror symmetry (4). This confirms the presence of destructive many-particle interference in the two symmetric scenarios alone.

A comparison of the theoretical and experimental results (see Fig. 3) yields reasonable agreement. Yet, for the mutually indistinguishable and intercycle distinguishable cases [see Figs. 3(a) and 3(b)], the simulation predicts a significantly lower degree of violation than measured. We attribute these deviations mainly to the following systematic errors and limitations of the model. (1) The experimental unitary reconstruction is subject to imprecisions and the theoretically assumed phase relations may not be perfectly realized (see Appendix B). (2) In addition to the substantial contributions from six generated photons on the statistics of four-photon outputs, there is also an eightphoton background (i.e., quadruple pair productions) that we estimate to comprise about $5 \%$ of all detected events. This background is not accounted for, due to the computational overhead. However, if we assume a uniform distribution of this eight-photon background among all fourfold outputs (suppressed and unsuppressed), we estimate that $\mathcal{D}_{\text {th }}$ would increase from initially 0.214 to 0.226 for the mutually indistinguishable scenario. (3) Inaccuracies in the estimated generation probabilities and losses before the unitary would lead to a different weighting of the contributions of the input states. That in turn may shift the probability distribution of the output events. (4) Finally, the indistinguishabilities of photons from the same pair, as well as of photons from different pairs, are estimated via HOM measurements (see Appendix F). We notice a drift in the indistinguishability during the measurement, which we correct for in the simulation, but this correction has its own precision limits (see Appendix E). Errors in the estimated indistinguishabilities lead to errors in the probability distribution of output events.

A fine-grained comparison of the experimental and theoretical data reveals that most bins in Fig. 3 agree within one or two standard deviations, while a few bins show a larger discrepancy. This is especially striking for the case of mutally distinguishable particles, shown in Fig. 3(d). As this scenario is governed by single-particle dynamics alone, we expect the imperfect unitary reconstruction [item (1) in the previous paragraph] to be the main source of systematic error for these events.
The optimal degree of suppression achievable in the experiment is mostly limited by the unwanted multiphoton background of the photon source. From a simulation excluding the six-photon background, we retrieve a theoretically predicted degree of suppression violation of 0.102 (compared to 0.214 with background) for the mutually indistinguishable case. It is possible to reduce this background by decreasing the SPDC pump power, which, however, also decreases the source brightness, leading to a longer integration time to achieve the same experimental quality of the output statistics. Since our integration time per configuration is $3-4$ days, this is only possible if one improves the transmission efficiency at the same time, e.g., via enhanced coupling from the fiber to the chip. Alternatively, one can replace the nondeterministic SPDC source by a quasideterministic source, e.g., by actively demultiplexing photons from a semiconductor quantum dot (QD) [8,52-54]. Resonantly excited semiconductor QDs feature almost zero multiphoton emission [55-57] and higher source brightness. Hence, they can be used to further optimize the suppression and, at the same time, increase the precision of the experiment.

\section{CONCLUSION}

In this work, we experimentally demonstrate that totally destructive many-particle interference (aka event suppression on output) does not necessarily require mutual indistinguishability of all involved particles. Instead, merely the symmetry of the input state matters, such that only particles occupying modes belonging to the same cycle of the underlying permutation symmetry must be indistinguishable. As a figure of merit, we use the degree of suppression violation $\mathcal{D}_{\text {exp }}$, which is significantly smaller for input states satisfying the symmetry required for the suppression as compared to input states violating this symmetry (and, thereby, destroying the necessary indistinguishability of those particles populating interfering manyparticle amplitudes which, by the cycle structure of the input state, contribute to the predicted suppression event). In order to simulate our experiment, we develop a general theoretical model designed to describe many-particle interference of photons with arbitrarily correlated spectral properties. Our model allows for the multifold occupation of input modes, incorporates quantitatively correct weights between the various emission orders in realistic SPDC photon sources with imperfections, and predicts the experimentally observed four-photon statistics with reasonable accuracy.

Our results clearly demonstrate that the observation of many-body suppression does not necessarily suffice to conclude that all involved constituents are mutually indistinguishable. This substantially restricts the range of symmetric scattering devices that can be used to unambiguously certify a boson sampler via fully destructive 
many-particle interference: In general, the output event suppression by multiparticle interference only certifies the symmetry of the input state with respect to a permutation of modes and the indistinguishability of those particles pertaining to subgroups identified by the diverse cycles of that very permutation. Only when this permutation consists of a single cycle of length $N$, as in the case of the $N \times N$ Fourier unitary $[12,21,27,35,39]$, or when a suitable set of permutation symmetries with shorter cycles is simultaneously satisfied, as is possible in Sylvester or hypercube unitaries $[23,24,30,36]$, does suppression suffice to certify the mutual indistinguishability of the particles.

\section{ACKNOWLEDGMENTS}

We acknowledge useful discussions with Stefan Frick. G.W., R.K., and J.M. acknowledge support from the Austrian Science Fund (FWF Projects No. I2562, No. P30459, and No. F7114). A.S. thanks the Deutsche Forschungsgemeinschaft (Grants No. SZ 276/9-2, No. SZ 276/12-1, No. SZ 276/20-1, No. SZ 276/21-1, and No. BL 574/13-1) and the Alfried Krupp von Bohlen and Halbach Foundation for financial support. M.L. acknowledges funding from the Marie Curie Actions within the Seventh Framework Programme for Research of the European Commission, under the Initial Training Network PICQUE, Grant No. 608062. C.D. acknowledges the Georg H. Endress foundation for financial support.

\section{APPENDIX A: MANY-PARTICLE INTERFERENCE FROM SPDC PHOTONS}

In this appendix, we present our theoretical model to describe many-particle interference experiments with photon pairs collected from a probabilistic multipair SPDC source. Note that our model is not limited to the present setup but can be used in a wide variety of experimental settings to calculate the input-output probabilities of pairwise correlated and partially distinguishable multiphoton states under any unitary transformation $U$. Similar considerations have been made for two photons fed into a Sylvester interferometer [30], where the authors consider fabrication errors in the unitary, multiphoton emission, and partial distinguishability of the photons. Here, our description generalizes this approach to multiple partially distinguishable photon pairs.

The model is divided into the following steps. We construct the $N$-photon input state (with, in general, $N>4$ ) consisting of $N / 2$ photon pairs obtained from SPDC and calculate the generation probabilities of these states in Sec. 1. The internal states of the particles are calculated from the product of reconstructed joint spectral amplitude (JSAs) of the emitted photon pairs. Loss before the unitary is modeled by extending the optical modes of the unitary with additional ancillary modes and unbalanced beam splitters, with lost photons being coupled into these ancillary modes (see Sec. 2). Afterward, for each so-constructed and loss-weighted input state, input-output probabilities are calculated according to our formalism presented in Sec. 3. This requires the matrix representation of the many-particle density operator, which accounts for partial distinguishability of the photons as calculated from the overlaps of the permuted internal states from Sec. 1.

In general, the relative phases between all input states must be taken into account in the calculation of the output probabilities, since these phases result in interference of the probability amplitudes corresponding to the different input states $[58,59]$. However, observing the time traces of recorded twofold coincidence counts shows that these relative phases fluctuate (see Appendix D) much faster than the integration time of the experiments. Therefore, the experimental data results from an incoherent mixture of these input states, such that we can consider an incoherent superposition of the input-output probabilities of all contributing input states weighted by their generation probabilities and losses. The resulting probabilities are then renormalized, accounting for the relative output transmissivities and detector efficiencies, which are obtained in the unitary reconstruction process (see Appendix B).

\section{Model of the SPDC source}

We consider the four-mode SPDC source (cf. Fig. 2) as two independent pulsed pair sources, which produce two quantum states, $\left|\Psi^{(A B)}\right\rangle$ and $\left|\Psi^{(C D)}\right\rangle$, in the two mode pairs of interest. The overall state reads [47]

$$
|\Psi\rangle=\left|\Psi^{(A B)}\right\rangle \otimes\left|\Psi^{(C D)}\right\rangle .
$$

The state of each parametric process can be written as a sum over the emission of $P$ photon pairs, with the state generated by the first source being [49]

$$
\left|\Psi^{(A B)}\right\rangle=c^{(A B)} \sum_{P=0}^{\infty} \frac{\left(p^{(A B)}\right)^{P / 2}}{P !} \sqrt{\mathcal{N}_{P}^{(A B)}}\left|\Psi_{P}^{(A B)}\right\rangle .
$$

Here, $c^{(A B)} \lesssim 1$ is a normalization constant and $\left(c^{(A B)}\right)^{2}$ $p^{(A B)} \approx p^{(A B)}$ is the pair-generation probability per pump pulse. The state of $P$ photon pairs produced in a pump pulse is given in a second quantization by an integral over the internal states of the particles, i.e., their frequencies [49],

$$
\begin{aligned}
\left|\Psi_{P}^{(A B)}\right\rangle= & \frac{1}{\sqrt{\mathcal{N}_{P}^{(A B)}}} \int d \vec{\omega} \Phi^{(A B)}\left(\omega_{1}, \omega_{1}^{\prime}\right) \cdots \Phi^{(A B)}\left(\omega_{P}, \omega_{P}^{\prime}\right) \\
& \times \hat{a}_{A}^{\dagger}\left(\omega_{1}\right) \hat{a}_{B}^{\dagger}\left(\omega_{1}^{\prime}\right) \cdots \hat{a}_{A}^{\dagger}\left(\omega_{P}\right) \hat{a}_{B}^{\dagger}\left(\omega_{P}^{\prime}\right)|0\rangle, \quad \text { (A3) }
\end{aligned}
$$

where $\Phi^{(A B)}\left(\omega, \omega^{\prime}\right)$ is the JSA of a single photon pair, $d \vec{\omega}=$ $d \omega_{1} d \omega_{1}^{\prime} \ldots d \omega_{P} d \omega_{P}^{\prime}$, and $\hat{a}_{A}^{\dagger}(\omega)$ is the creation operator of 
a photon with frequency $\omega$ in mode $A$. The normalization coefficient $\mathcal{N}_{P}^{(A B)}$ is dictated by the commutation rules of the ladder operators and can be calculated via a summation over all intramode permutations:

$$
\begin{aligned}
\mathcal{N}_{P}^{(A B)}= & \int d \vec{\omega}\left(\Phi^{(A B)}\left(\omega_{1}, \omega_{1}^{\prime}\right) \cdots \Phi^{(A B)}\left(\omega_{P}, \omega_{P}^{\prime}\right)\right)^{*} \\
& \times \sum_{\pi, \sigma \in S_{P}} \Phi^{(A B)}\left(\omega_{\pi(1)}, \omega_{\sigma(1)}^{\prime}\right) \cdots \Phi^{(A B)}\left(\omega_{\pi(P)}, \omega_{\sigma(P)}^{\prime}\right) .
\end{aligned}
$$

Here, $S_{P}$ is the symmetric group of $P$ elements (corresponding to the $P$ particles in each of the two modes). Equation (A2) can be interpreted as an interpolation between a two-mode squeezed vacuum state $\left(\mathcal{N}_{P}^{(A B)}=P !^{2}\right)$ and purely accidental multipair generation $\left(\mathcal{N}_{P}^{(A B)}=P\right.$ !). The former arises from an uncorrelated JSA $\left[\Phi\left(\omega, \omega^{\prime}\right)=\phi(\omega) \phi\left(\omega^{\prime}\right)\right]$ and a maximal contribution of stimulated emission and leads to perfect heralded HOM visibility between photons from different pairs [47], while the latter corresponds to a maximally correlated JSA $\left[\Phi\left(\omega, \omega^{\prime}\right)=\delta\left(\omega+\omega^{\prime}-\omega_{\mathrm{p}}\right) \Phi\left(\omega, \omega^{\prime}\right)=\right.$ $\Phi\left(\omega, \omega-\omega_{\mathrm{p}}\right)$ with the pump frequency $\left.\omega_{\mathrm{p}}\right]$ and purely spontaneous emission, producing zero HOM visibility. Note that the difference between these two extremes vanishes in the case of single-pair emission, i.e., $P=1$.

The probability of the first source generating the state $\left|\Psi_{P}^{(A B)}\right\rangle$ of $P$ pairs is derived from Eq. (A2):

$$
p_{P}^{(A B)}=\left(c^{(A B)}\right)^{2} \frac{\left(p^{(A B)}\right)^{P}}{P !^{2}} \mathcal{N}_{P}^{(A B)} .
$$

The joint probability of generating $P$ pairs in channels $A$ and $B$, and $Q$ pairs in channels $C$ and $D$ is then

$$
p_{P, Q}=\left(c^{(A B)} c^{(C D)}\right)^{2}\left(p^{(A B)}\right)^{P}\left(p^{(C D)}\right)^{Q} \frac{\mathcal{N}_{P}^{(A B)} \mathcal{N}_{Q}^{(C D)}}{P !^{2} Q !^{2}} .
$$

From a preliminary characterization of the source, we obtain $p^{(A B)}=0.026$ and $p^{(C D)}=0.033$ (see Appendix F). In order to calculate $\mathcal{N}_{P}^{(A B),(C D)}$, we construct JSAs $\Phi^{(A B),(C D)}\left(\omega, \omega^{\prime}\right)$ that reproduce our experimentally measured HOM and heralded HOM visibilities (see Appendix E). From this, we obtain numeric values of $\mathcal{N}_{2}^{(A B)} \approx \mathcal{N}_{2}^{(C D)} \approx 3.18$ and $\mathcal{N}_{3}^{(A B)} \approx \mathcal{N}_{3}^{(C D)} \approx 21.65$. Note that in order to account for the distinguishability between the particles induced by different times of arrival in the experimentally investigated cases of intercycle distinguishable and intracycle distinguishable particles, we include an additional phase $e^{i \omega \tau}$ with a sufficiently large temporal delay $\tau$ between the SPDC source channels. With Eq. (A6), we calculate the generation probability of states up to six photons, which is summarized in Table II.
TABLE II. All states consisting of up to six photons that possibly lead to a fourfold coincidence after evolving under the unitary transformation $U_{\text {exp }} \vec{R}_{(A, B, C, D)}$ is the mode occupation list of the four SPDC channels, $N$ is the number of photons in that state, $\vec{R}_{J_{x}}$ is the mode occupation list for the $n=7$ input modes of the $J_{x}$ unitary (excluding the additional unoccupied ancillary input modes), $p_{\text {gen }}$ is the generation probability calculated via Eq. (A6), and $p_{\text {gen,norm }}$ is the generation probability normalized to all listed states. Note that despite the very low generation probabilities of the six-photon states, they contribute significantly to the final output probability, due to a combinatorial advantage in the lossy setup.

\begin{tabular}{lcccc}
\hline \hline$\vec{R}_{(A, B, C, D)}$ & $N$ & $\vec{R}_{J_{x}}$ & $p_{\text {gen }}$ & $p_{\text {gen,norm }}$ \\
\hline$(2,2,0,0)$ & 4 & $\vec{R}_{1}$ & $4.93 \times 10^{-4}$ & 0.230 \\
$(1,1,1,1)$ & 4 & $\vec{R}_{2}$ & $7.88 \times 10^{-4}$ & 0.368 \\
$(0,0,2,2)$ & 4 & $\vec{R}_{3}$ & $7.97 \times 10^{-4}$ & 0.372 \\
$(3,3,0,0)$ & 6 & $(3,0,0,0,0,0,3)$ & $0.10 \times 10^{-4}$ & 0.004 \\
$(2,2,1,1)$ & 6 & $(2,0,1,0,1,0,2)$ & $0.16 \times 10^{-4}$ & 0.007 \\
$(1,1,2,2)$ & 6 & $(1,0,2,0,2,0,1)$ & $0.20 \times 10^{-4}$ & 0.010 \\
$(0,0,3,3)$ & 6 & $(0,0,3,0,3,0,0)$ & $0.20 \times 10^{-4}$ & 0.009 \\
\hline \hline
\end{tabular}

We find a $32 \times$ enhanced generation probability of fourphoton states compared to six-photon states. Despite this seemingly low rate of created six-photon states, they nevertheless contribute significantly to the measured counting statistics due a combinatorial advantage over fourphoton states in the lossy setup. To yield a four-photon coincidence from an initial six-photon state, up to two photons may be lost before detection. There are $\left(\begin{array}{l}6 \\ 2\end{array}\right)=$ 15 possible combinations of lost photons, which significantly increases the relative weight of six-photon states in the lossy setup. From the simulation, we predict that $33.5 \%-35.0 \%$ (depending on the measured scenario) of all registered nonbunching output events originate from an initial six-photon creation.

\section{Photon loss}

Loss processes before the fabricated $J_{x}$ unitary are summarized in the channel transmission factors $\eta_{j}$ (for their values and the method of characterization, see Appendix F). Their combined impact on the dynamics can be modeled by extending $U_{\text {exp }}$ with seven uncoupled ancillary modes and prepending to it a unitary of seven unbalanced beam splitters (one for each mode) feeding the lost photons into these ancillary modes with probability $1-\eta_{j}$. This extended unitary $\mathcal{U}$ is then used in the subsequent calculations.

\section{Partial distinguishability of correlated photon pairs}

Let us consider a state of $N$ partially distinguishable photons, which occupy external states $|\vec{E}\rangle=\left|E_{1}, \ldots, E_{N}\right\rangle$ of an $n$-mode scattering network with unitary $\mathcal{U}$. The $\mathrm{N}$-particle state is determined by its mode occupation list 
$\vec{R}=\left(R_{1}, \ldots, R_{n}\right)$ or, equivalently, by its mode assignment list $\vec{E}=\left(E_{1}, \ldots, E_{N}\right)$, where $E_{\alpha}$ is the mode occupied by the $\alpha$ th particle [33]. The internal state of the particles is modeled by frequencies $|\vec{\omega}\rangle=\left|\omega_{1}, \ldots, \omega_{N}\right\rangle$, yielding the combined state

$$
|\vec{E}\rangle \otimes|\vec{\omega}\rangle=\left|E_{1}, \ldots, E_{N}\right\rangle \otimes\left|\omega_{1}, \ldots, \omega_{N}\right\rangle .
$$

The combined state of a correlated photon pair from SPDC in the mode pair $A, B$ is described by an integral over the internal states of the particles, which are correlated according to the JSA $\Phi\left(\omega_{1}, \omega_{2}\right)$. In the first quantization without symmetrization, the combined state is given by

$$
\int d \omega_{1} d \omega_{2} \Phi\left(\omega_{1}, \omega_{2}\right)|A, B\rangle\left|\omega_{1}, \omega_{2}\right\rangle .
$$

To correctly include the exchange symmetry of bosons, the two-photon state needs to be symmetric under exchange of particles. After the required symmetrization, the state of one created photon pair reads

$$
\left|\Psi_{1}^{(A B)}\right\rangle=\int d \omega_{1} d \omega_{2} \Phi\left(\omega_{1}, \omega_{2}\right)|\vec{R}: \vec{\omega}\rangle,
$$

where $|\vec{R}: \vec{\omega}\rangle=\left(|A, B\rangle\left|\omega_{1}, \omega_{2}\right\rangle+|B, A\rangle\left|\omega_{2}, \omega_{1}\right\rangle\right) / \sqrt{2}$ and the normalization condition of the JSA is $\int d \omega_{1} \mathrm{~d} \omega_{2}$ $\left|\Phi\left(\omega_{1}, \omega_{2}\right)\right|^{2}=1$.

Next, we consider a state of $P$ photon pairs emitted from the first source into modes $A$ and $B$, as well as $Q$ photon pairs emitted from a second source into modes $C$ and $D$. The total number of photons is $N=2(P+Q)$. We can describe the state by symmetrizing the expression

$$
\int d \vec{\omega} \Phi(\vec{\omega})|\vec{E}\rangle|\vec{\omega}\rangle
$$

with the total JSA given by the product of JSAs corresponding to all photon pairs,

$$
\Phi(\vec{\omega})=\prod_{i=1}^{P} \Phi^{(A B)}\left(\omega_{2 i-1}, \omega_{2 i}\right) \prod_{j=P+1}^{P+Q} \Phi^{(C D)}\left(\omega_{2 j-1}, \omega_{2 j}\right),
$$

as well as

$$
|\vec{E}\rangle=|\underbrace{A, B \ldots, A, B}_{A, B ; P \text { times }}, \underbrace{C, D \ldots, C, D}_{C, D ; Q \text { times }}\rangle,
$$

and $d \vec{\omega}=d \omega_{1} \ldots d \omega_{N}$. Symmetrization of the above equation yields the state

$$
\left|\Psi_{P, Q}\right\rangle \propto \sum_{\pi \in S_{N}}\left|\vec{E}_{\pi}\right\rangle \otimes \int d \vec{\omega} \Phi(\vec{\omega})\left|\vec{\omega}_{\pi}\right\rangle,
$$

where we use the shorthands $\left|\vec{E}_{\pi}\right\rangle=\left|E_{\pi(1)}, \ldots, E_{\pi(N)}\right\rangle$ and $\left|\vec{\omega}_{\pi}\right\rangle=\left|\omega_{\pi(1)}, \ldots, \omega_{\pi(N)}\right\rangle$. The calculation of Eq. (A10) can be simplified by reducing the summation over all permutations $S_{N}$ to a summation over permutations leading to inequivalent external states $\left|\vec{E}_{\pi}\right\rangle$, that is, to permutations that only permute particles across different modes. To this end, one can decompose $\pi \in S_{N}$ as $\pi=\xi \mu$ with $\xi \in S_{\vec{R}}$ and $\mu \in \Sigma$. Here, $S_{\vec{R}}=S_{R_{1}} \otimes \cdots \otimes S_{R_{N}}$ is a Young subgroup of $S_{N}$ and $\Sigma$ is the right transversal of $S_{\vec{R}}$ in $S_{N}$ $[18,39]$. This results in

$$
\left|\Psi_{P, Q}\right\rangle \propto \sum_{\mu \in \Sigma, \xi \in S_{\vec{R}}}\left|\vec{E}_{\xi \mu}\right\rangle \otimes \int d \vec{\omega} \Phi(\vec{\omega})\left|\vec{\omega}_{\xi \mu}\right\rangle .
$$

We utilize $\left|\vec{E}_{\xi \mu}\right\rangle=\left|\vec{E}_{\mu}\right\rangle$ and write $\vec{\omega}$ as $\vec{w}_{\xi^{\prime}}$ with $\xi^{\prime} \in S_{\vec{R}}$, and $\vec{w} \in X_{\vec{w}}=\left\{\vec{w}_{\xi} \mid \xi \in S_{\vec{R}}\right\}$ (such that $\{\vec{\omega}\}=\cup_{\vec{w}} X_{\vec{w}}$ and $\left.\int d \vec{\omega}=\int d \vec{w} \sum_{\xi^{\prime} \in S_{\vec{R}}}\right)$. This yields

$$
\left|\Psi_{P, Q}\right\rangle \propto \sum_{\mu \in \Sigma}\left|\vec{E}_{\mu}\right\rangle \otimes \int d \vec{w} \sum_{\xi, \xi^{\prime} \in S_{\vec{R}}} \Phi\left(\vec{w}_{\xi^{\prime}}\right)\left|\vec{w}_{\xi^{\prime} \xi \mu}\right\rangle .
$$

Since $S_{\vec{R}}$ forms a group, we can substitute $\xi^{\prime} \xi=\xi^{\prime \prime}$ and sum over $\xi^{\prime \prime}$ instead of $\xi^{\prime}$ :

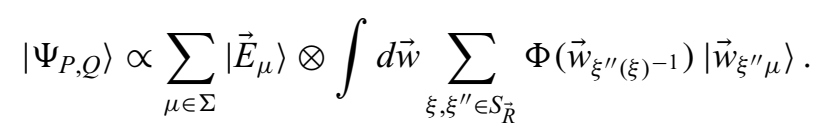

Next, we use the same trick as above and write $\vec{w}_{\xi^{\prime \prime}}$ as $\vec{\omega}$, with $\int d \vec{w} \sum_{\xi^{\prime \prime} \in S_{\vec{R}}}=\int d \vec{\omega}$, and instead of summing over $\xi$, we sum over $(\xi)^{-1}$ (this substitution can be done since $S_{\vec{R}}$ forms a group), which results in

$$
\left|\Psi_{P, Q}\right\rangle \propto \sum_{\mu \in \Sigma}\left|\vec{E}_{\mu}\right\rangle \otimes \int d \vec{\omega}\left(\sum_{\xi \in S_{\vec{R}}} \Phi\left(\vec{\omega}_{\xi}\right)\right)\left|\vec{\omega}_{\mu}\right\rangle .
$$

We identify the not-yet-normalized coefficients (in the parentheses):

$$
\bar{C}(\vec{\omega})=\sum_{\xi \in S_{\vec{R}}} \Phi\left(\vec{\omega}_{\xi}\right)
$$

Normalization of Eq. (A14) then leads to [18,39]

$$
\left|\Psi_{P, Q}\right\rangle=\frac{1}{\sqrt{R}} \sum_{\mu \in \Sigma}\left|\vec{E}_{\mu}\right\rangle \otimes\left|\Omega_{\mu}\right\rangle,
$$

where $R=N ! /\left|S_{\vec{R}}\right|=N ! /(P ! Q !)^{2}$ and

$$
\left|\Omega_{\mu}\right\rangle=\int d \vec{\omega} C(\vec{\omega})\left|\vec{\omega}_{\mu}\right\rangle
$$


The internal state coefficients $C(\vec{\omega})$ take into account the possible orderings within a mode and are calculated from

$$
C(\vec{\omega})=\frac{\sum_{\xi \in S_{\vec{R}}} \Phi\left(\vec{\omega}_{\xi}\right)}{\sqrt{\int \mathrm{d} \vec{\omega}^{\prime}\left|\sum_{\xi \in S_{\vec{R}}} \Phi\left(\vec{\omega}^{\prime} \xi\right)\right|^{2}}},
$$

with the normalization factor in the denominator such that $\int d \vec{\omega}|C(\vec{\omega})|^{2}=1$

The many-particle state (A16) describes the initial state of our experiment. Since the unitary transformation $\mathcal{U}$ and the measurement of the output-mode occupation of the particles do not act upon the internal degrees of freedom of the particles, we can trace them out, resulting in the reduced external many-particle state

$$
\begin{aligned}
\rho_{E} & =\operatorname{Tr}_{I}\left(\left|\Psi_{P, Q}\right\rangle\left\langle\Psi_{P, Q}\right|\right) \\
& =\sum_{\mu, v \in \Sigma}\left[\rho_{E}\right]_{\mu, v}\left|\vec{E}_{\mu}\right\rangle\left\langle\vec{E}_{\nu}\right|,
\end{aligned}
$$

with

$$
\left[\rho_{E}\right]_{\mu, \nu}=\frac{1}{R}\left\langle\Omega_{\nu} \mid \Omega_{\mu}\right\rangle .
$$

The transition probability to obtain the output-mode occupation $\vec{S}$ from the input-mode occupation $\vec{R}$ is then

obtained by [39]

$$
p_{\vec{R} \rightarrow \vec{S}}=S \sum_{\mu, v \in \Sigma}\left[\rho_{E}\right]_{\mu, \nu} \prod_{\alpha=1}^{N} \mathcal{U}_{F_{\alpha}, E_{\mu(\alpha)}} \mathcal{U}_{F_{\alpha}, E_{\nu}(\alpha)}^{*},
$$

where $S=N ! /\left|S_{\vec{S}}\right|,\left|S_{\vec{S}}\right|=\prod_{j=1}^{n} S_{j}$ ! and $\vec{F}$ is the outputmode assignment list (defined similar to the input-mode assignment list $\vec{E}$ ). Using Eq. (A21), we can calculate all relevant input-output probabilities for each input state listed in Table II. Note that an output fourfold coincidence can originate from any of the four-photon input states $\vec{R}_{1}$, $\vec{R}_{2}, \vec{R}_{3}$, with no photon being lost. However, fourfold coincidences can also arise from a six-photon input state, with up to two photons ending up in ancillary modes and/or up to two photons lost after the unitary and/or up to three photons occupying the same output mode (collision event). All these cases are subsumed as the six-photon background in Fig. 3.

\section{APPENDIX B: WAVEGUIDE FABRICATION AND UNITARY CHARACTERIZATION}

We fabricate the waveguide chip implementing the $J_{x}$ unitary in fused silica (Corning 7980, ArF grade) using the femtosecond-laser direct-write approach [43]. Due to nonlinear absorption, the transparent material is modified within the focal region, producing a local increase of the
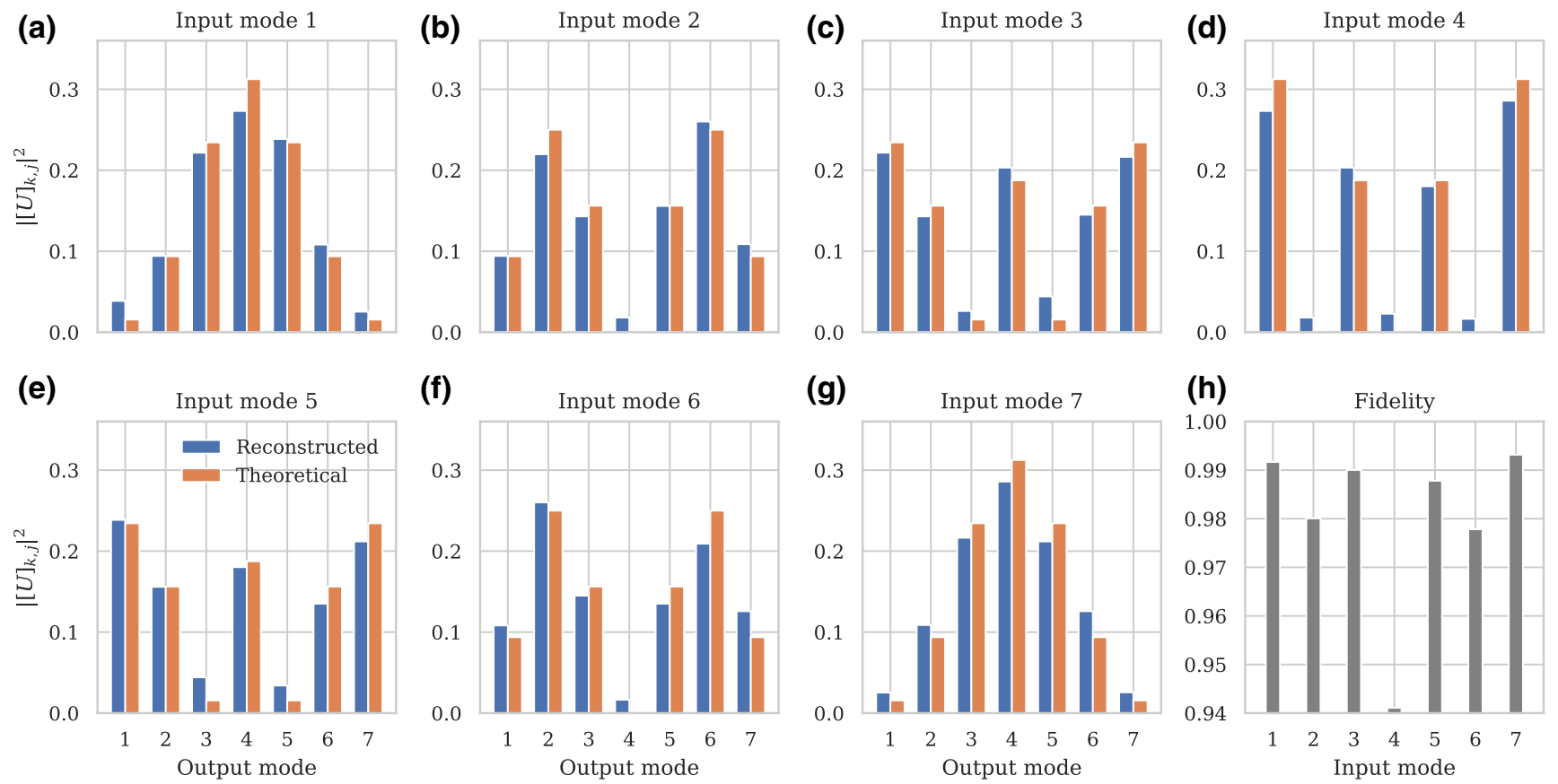

FIG. 4. The reconstructed magnitudes of the $J_{x}$ unitary. (a)-(g) We launch single photons into each input mode of the waveguide structure and measure the relative output intensity in each output mode. From this, we reconstruct $\left|\left[U_{\text {recon }}\right]_{k, j}\right|^{2}$, following the algorithm from Ref. [61]. (h) The fidelities $F_{j}=\left(\sum_{k=1}^{7} \sqrt{\left|\left[U_{\text {recon }}\right]_{k, j}\right|^{2} \times\left|\left[U^{J}\right]_{k, j}\right|^{2}}\right)^{2}$ of the reconstructed magnitudes, which benchmark how well the physical unitary agrees with the ideal $J_{x}$ unitary. 
refractive index. A Coherent RegA 9000 amplifier seeded by a Mira Ti:Sa femtosecond-laser oscillator is used. After amplification, the pulses with 150 -fs pulse duration centered at $800 \mathrm{~nm}$ have an energy of $450 \mathrm{~nJ}$ at a repetition rate of $100 \mathrm{kHz}$. The waveguides are permanently inscribed in the bulk while moving the sample at a constant speed of $60 \mathrm{~mm} \mathrm{~min}^{-1}$ (high-precision positioning stages ALS 130 , Aerotech Inc., with a positioning error of $\pm 0.1 \mu \mathrm{m}$ ). The beam is focused with a $20 \times$ objective, producing a mode field diameter of the guided mode of the order of $18 \mu \mathrm{m} \times 20 \mu \mathrm{m}$ at $815 \mathrm{~nm}$. The fan-in and fan-out sections are arranged in a three-dimensional geometry to permit coupling to fiber arrays with a standard spacing of $127 \mu \mathrm{m}$ while minimizing crosstalk. These sections are written over three times to mitigate bending losses and to reduce the mode-field diameter at the end facets to about $10 \mu \mathrm{m} \times 14 \mu \mathrm{m}$ for improved mode matching to optical fibers [60].

Prior to the experiment, we characterize the unitary using single photons. The magnitudes of the experimentally reconstructed unitary matrix and the ideal $J_{x}$ unitary are compared in Fig. 4. We reconstruct the unitary by sending single photons from the SPDC source into each input mode separately. For each input, we monitor the singlephoton count rate in each of the seven output modes using APDs. By performing a least-squares optimization, we reconstruct the magnitude squares $\left|\left[U_{\text {recon }}\right]_{k, j}\right|^{2}$ according to the procedure in Ref. [61]. From these data, we additionally retrieve the relative output transmissivities (including detector efficiencies), which we use in our simulation of the four-photon output probabilities (theory bars in Fig. 3). The phases of the matrix elements, $\left[\operatorname{Arg}\left(U_{\text {recon }}\right)\right]_{k, j}$, are not reconstructed, as we expect that the fabricated structure closely matches the ideal phases of the $J_{x}$-lattice. According to our experience with similar waveguide structures, the propagation constant of the laser-written waveguides (which ultimately determines these phases) is much less sensitive to fabrication errors than the coupling rate between neighboring waveguides (which only influences the magnitudes of the unitary matrix). A two-photon interference experiment conducted on the sample supports our hypothesis (see Fig. 7), since its results agree well with the theoretical predictions, which are based on the ideal phases of the $J_{x}$ unitary. Therefore, we model the matrix elements of the unitary probed in our experiment as $\left[U_{\exp }\right]_{k, j}=$ $\left|\left[U_{\text {recon }}\right]_{k, j}\right| \times e^{i\left[\operatorname{Arg}\left(U^{J}\right)\right]_{k, j}}$. For a more refined model, the unitary phases could be retrieved experimentally with the techniques described in Refs. [62-65].

\section{APPENDIX C: PROCEDURE TO TEMPORALLY OVERLAP THE SPDC CHANNELS}

We adjust the optical delays between the four inputs to the unitary by performing two-photon HOM-type measurements on the waveguide structure. First, we launch channels $C$ and $D$ into input modes 3 and 5, respectively. We monitor coincidences between output modes 1 and 4, while scanning the optical delay stage. The corresponding output state is almost perfectly suppressed for indistinguishable photons, while showing a reasonably high output probability for distinguishable photons. In the same fashion, we optimize source channel $B$ relative to channel $D$, and thereafter channel $A$ relative to channel $B$, while monitoring for each delay scanning multiple output combinations at the same time that exhibit good contrast in the detected coincidence rate between distinguishable and indistinguishable photons.

\section{APPENDIX D: PHASE FLUCTUATION FROM TWOFOLD COINCIDENCE DATA}

In general, one needs to take the relative phases of all contributing input states into account in the calculation of the final output probability distribution. By applying and averaging over a series of phases in one of the input modes, one can realize a mixed state, which allows for simpler addition of probabilities. This can be realized via a combination of quarter- and half-wave plates, oriented such that the input state is kept unchanged except for an additional phase factor [38,59]. In our experiment, the mixed state is automatically obtained via time integration, as the relative phases fluctuate rapidly during the measurement. This can be shown by looking at the time traces of twofold coincidence counts for the scenario of mutually indistinguishable photons. These time traces are recorded parallel to the fourphoton coincidences of the main experiment. Figure 5(a) depicts the coincidence rate of two output states with approximately equal contributions from input states $\vec{R}_{4}=$ $(0,0,1,0,1,0,0)$ and $\vec{R}_{5}=(1,0,0,0,0,0,1)$ (see the two rightmost states in Fig. 7). The coincidence rates fluctuate strongly from 50 to $250 \mathrm{~Hz}$, while the single-photon count rates remain approximately constant [Fig. 5(b)]. This suggests that the relative phase of the contributing inputs fluctuated, e.g., through temperature drifts of the optical fibers from the source to the unitary.

\section{APPENDIX E: ESTIMATION OF AVERAGE HOM VISIBILITY FROM TWOFOLD COINCIDENCES AND JSA RECONSTRUCTION}

For the investigated scenario of mutually indistinguishable particles, we observe a slow change in the recorded twofold coincidence counts over time [compare Figs. 5(c) and 5(d)]. This effect is most pronounced for output states, which receive contributions mostly from the input state $\vec{R}_{4}$. We attribute this drift to relative optical-path-length changes in the pertinent channels (here, $C$ and $D$ ) of the setup from the SPDC source to the $J_{x}$ unitary, which leads to a degradation of the indistinguishability from the initially optimized situation. This may arise from the 

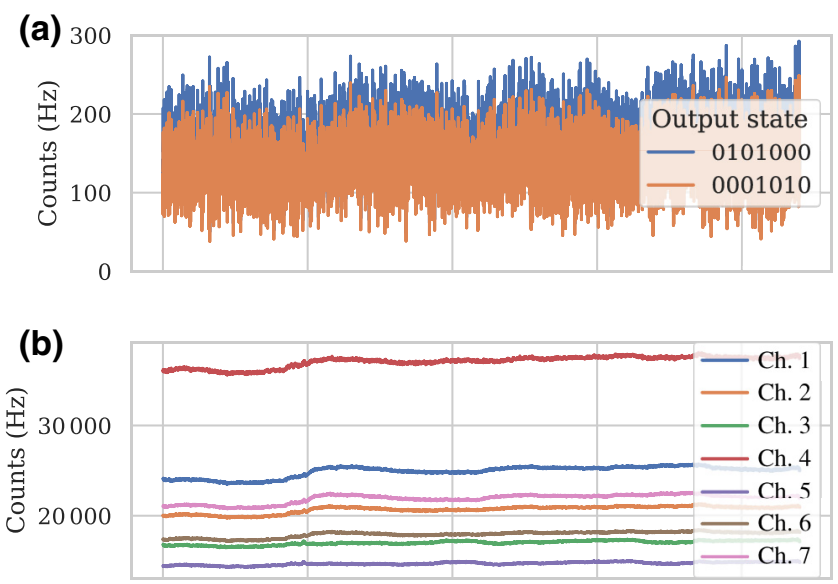

(c)

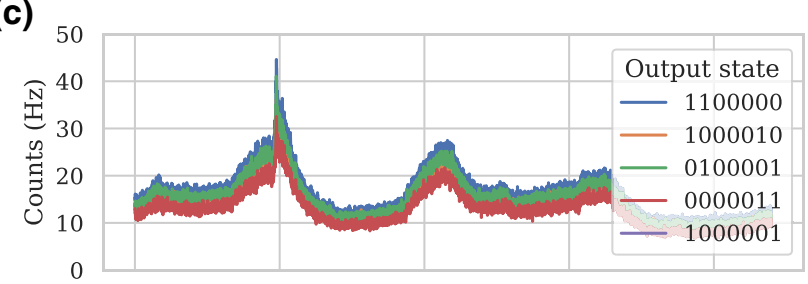

(d)

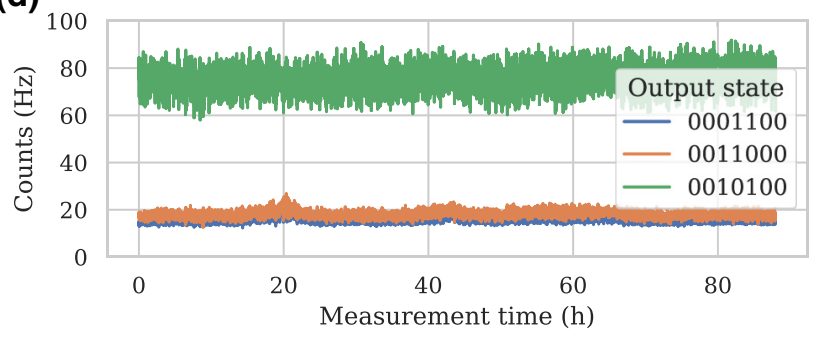

FIG. 5. Selected twofold coincidence counts and singlephoton count rates during the measurement of the mutually indistinguishable particle scenario. Counts are averaged over an interval of $60 \mathrm{~s}$ for a total measurement time of $88 \mathrm{~h}$. (a) The time traces of two output states that have approximately equal contributions from input state $\vec{R}_{4}=(0,0,1,0,1,0,0)$ and $\vec{R}_{5}=(1,0,0,0,0,0,1)$ according to the theoretical predictions. The observed coincidence rate fluctuates heavily, suggesting that the relative phase of these two contributions fluctuates. (b) The time traces of the single-photon count rates of all seven output channels. (c) Selected time traces of output states that are dominated by contributions of input state $\vec{R}_{4}$. These output states are ideally suppressed according to the suppression law. We attribute the slow drift in the observed coincidence rates to a change in the indistinguishability of the photons caused by changes of the optical path length. Hardly any fast fluctuations in the coincidence rate are observed, since mostly one input state contributes. (d) Selected time traces of the output states, which are dominated by contributions of input state $R_{5}$. Neither strong fast fluctuations nor significant slow drifts of the coincidence rates are observed.

asymmetric response to temperature changes of the freespace delay in channel $C$ and the fiber in channel $D$ and it changes the output probability distribution (counting statistics), e.g., it may increase the probability of observing an ideally suppressed output event due to a degrading of the indistinguishability of the particles.

We estimate the time-averaged degraded indistinguishability of photons from the same pair from the total experimental twofold coincidence counts, which we collect in parallel to the fourfold coincidences of the main experiment. We compare the experimentally obtained output distribution of all nonbunching two-photon events with the simulated output distribution for varying indistinguishabilities $V_{A B}$ and $V_{C D}$, i.e., indistinguishability between photons in channels $A$ and $B$, as well as channels $C$ and $D$. Additionally, we keep the indistinguishability between photons originating from different pairs at a fixed value of $59.1 \%$ (retrieved from a separate heralded HOM measurement, see Appendix F).

For each simulated output distribution, we calculate the fidelity according to

$$
F=\left(\sum_{i} \sqrt{p_{\mathrm{exp}, i} p_{\mathrm{sim}, i}}\right)^{2},
$$

with the sum running over all nonbunching two-photon outputs. We consider input states $\vec{R}_{4}$ and $\vec{R}_{5}$, as well as a background of four- and six-photon states, where two and four photons are lost before the unitary, respectively (these are the dominant contributions under the considerations of losses). The background resulting from initial states of four and six photons depends on the indistinguishability between photons from different pairs and contributes

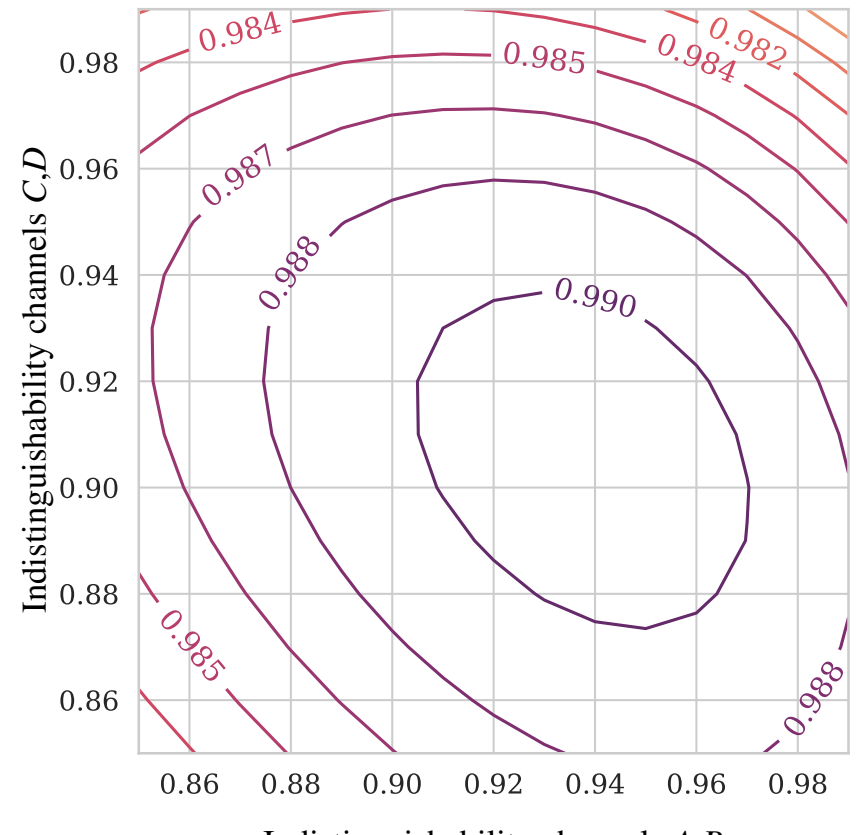

Indistinguishability channels $A, B$

FIG. 6. The calculated fidelity for an output probability distribution of all twofold nonbunching coincidence events as a function of the indistinguishabilities $V_{A B}$ and $V_{C D}$. 


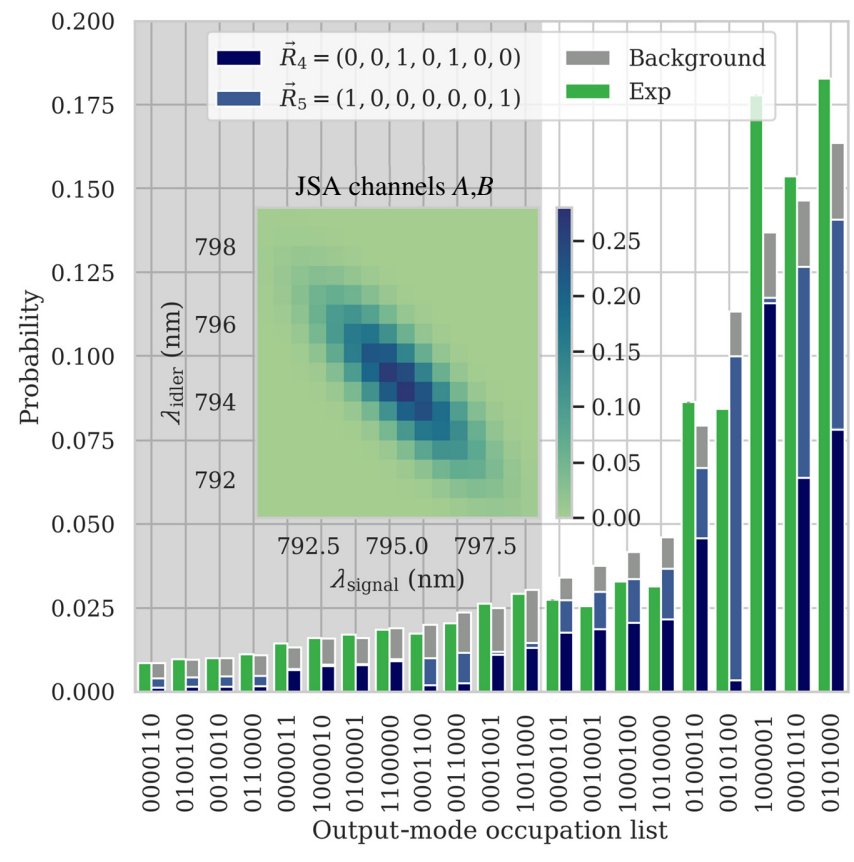

FIG. 7. The output probability distribution of all twofold nonbunching coincidence events. For the simulated result, we use optimized time-averaged indistinguishabilities of $V_{A B}=0.94$ and $V_{C D}=0.90$. The fidelity is $F=0.991$. The experimental and simulated degree of suppression violation are $D_{\exp }=0.198$ and $D_{\text {sim }}=0.202$, respectively. The experimental error bars from the counting statistics are too small to be visible. The inset shows the discretized JSA of channels $A$ and $B$ (the JSA of channels $C$ and $D$ is similar).

around $22.4 \%$ to the measured final output events. As shown in Fig. 6, the fidelity reaches its maximum value for indistinguishabilities of $V_{A B}=0.94$ and $V_{C D}=0.90$. These values should therefore best represent the timeaveraged indistinguishabilities in the experiment. For the above estimated time-averaged indistinguishabilities, the normalized output distribution of all nonbunching twofold coincidence events is plotted in Fig. 7. It reaches a fidelity of $F=0.991$.

We finally calculate a JSA that reproduces the measured and estimated indistinguishabilities by following the procedure in Ref. [66]. First, a phase-matching function is calculated in the paraxial approximation of the involved spatial modes of the pump laser and the photon-collecting fibers. We apply a Gaussian pump spectral envelope and spectral-filter functions (3-nm FWHM bandwidth, as in the setup) to obtain a discretized JSA on a frequency grid of size $17 \times 17$. The JSAs of SPDC channels $A$ and $B$ are plotted in the inset of Fig. 7.

Instead of modeling the reduction of indistinguishability by a path-length drift directly in the time domain, we mimic this effect by mutually shifting the central transmission frequencies of the filters (thus reducing spectral instead of temporal overlaps). The estimated time-averaged indistinguishabilities are reproduced with an up-converted pump spectral width of $0.4 \mathrm{~nm}$ FWHM, as well as pairwise filter offsets of $0.625 \mathrm{~nm}$ between channels $A$ and $B$ and $0.8 \mathrm{~nm}$ between channels $C$ and $D$. The internal state of multiple pairs is then calculated as a tensor product of the two-photon JSAs [cf. Eq. (A9)].

\section{APPENDIX F: SOURCE PARAMETERS AND PHOTON LOSS}

To properly simulate the source and subsequent channel losses in the experiment, we need to estimate the pairgeneration probability and the collection efficiencies of the source, as well as the indistinguishabilities of photons from different channels.

A preliminary characterization of the source allows us to obtain the pair-generation probability and the collection efficiencies of the four source channels. We attach each source channel directly to an APD, which results in typical single-photon count rates of $160-370 \mathrm{kHz}$ at a pump power of approximately $150 \mathrm{~mW}$. Additionally, we measure coincidence rates of $31 \mathrm{kHz}$ between channels $A$ and $B$, as well as $25 \mathrm{kHz}$ between channels $C$ and $D$. From these rates, we reconstruct pair-generation probabilities per pump pulse of $p^{(A B)}=2.6 \%$ and $p^{(C D)}=3.3 \%$ for channels $A$ and $B$, and channels $C$ and $D$, respectively. Additionally, we obtain collection efficiencies of $19 \%, 18 \%, 10 \%$, and $22 \%$ for the four channels $A-D$. In the source characterization, we use detectors with a detection efficiency of $68 \%$. The collection efficiencies are corrected for this detection efficiency.

These channel-collection efficiencies, as well as the incoupling efficiencies to the seven input modes of the unitary, which include fiber-to-chip coupling and propagation loss on the chip, are combined to input-mode transmissivity factors $\eta_{j}$ for $j=1, \ldots, 7$. We extract the incoupling efficiencies from the unitary-reconstruction data (cf. Appendix B), comparing the total transmissivity across all output modes for the relevant input modes and assuming an on-chip propagation loss of $0.5 \mathrm{~dB} / \mathrm{cm}$ in the central interaction region and $0.3 \mathrm{~dB} / \mathrm{cm}$ in the fan-out section of the waveguide structure (the loss is reduced in the fan-out section due to a stronger mode confinement). We retrieve incoupling efficiencies of $31 \%, 38 \%, 52 \%$, and $40 \%$ for the four occupied input modes 1, 3, 5, and 7 in the experiment, respectively. Fiber-to-chip coupling is mainly limited by mode mismatch at the interface (calculated as $55 \%$ from the mode overlap) and could be further improved by using thermally expanded core fibers [67]. From this, we calculate the input-mode transmissivity factors $\eta_{1}=0.058$, $\eta_{3}=0.036, \eta_{5}=0.114$, and $\eta_{7}=0.069$.

We perform a heralded HOM-interference experiment on a fiber beam splitter, where we interfere photons from channels $B$ and $D$, using $A$ and $C$ as additional heralding channels [47]. From the experiment, we attain an indistinguishability of $59.1 \%$ for photons originating from 
different pairs. In a standard HOM experiment with photons from the same pair, we retrieve maximal visibilities of $96.1 \%$ for source channels $A$ and $B$ and $98.2 \%$ for source channels $C$ and $D$. In the actual experiment, these indistinguishabilities vary over time, probably due to opticalpath-length drifts, which reduces the temporal overlap of photons from different channels (see Appendix E).

[1] E. Knill, R. Laflamme, and G. J. Milburn, A scheme for efficient quantum computation with linear optics, Nature 409 , 46 (2001).

[2] D. Bouwmeester, J. W. Pan, K. Mattle, M. Eibl, H. Weinfurter, and A. Zeilinger, Experimental quantum teleportation, Nature 390, 575 (1997).

[3] B. Hensen, H. Bernien, A. E. Dreaú, A. Reiserer, N. Kalb, M. S. Blok, J. Ruitenberg, R. F. Vermeulen, R. N. Schouten, C. Abellán, W. Amaya, V. Pruneri, M. W. Mitchell, M. Markham, D. J. Twitchen, D. Elkouss, S. Wehner, T. H. Taminiau, and R. Hanson, Loophole-free Bell inequality violation using electron spins separated by 1.3 kilometres, Nature 526, 682 (2015).

[4] M. A. Broome, A. Fedrizzi, S. Rahimi-Keshari, J. Dove, S. Aaronson, T. C. Ralph, and A. G. White, Photonic boson sampling in a tunable circuit, Science 339, 794 (2013).

[5] J. B. Spring, B. J. Metcalf, P. C. Humphreys, W. S. Kolthammer, X.-M. Jin, M. Barbieri, A. Datta, N. ThomasPeter, N. K. Langford, D. Kundys, J. C. Gates, B. J. Smith, P. G. R. Smith, and I. A. Walmsley, Boson sampling on a photonic chip, Science 339, 798 (2013).

[6] M. Tillmann, B. Dakić, R. Heilmann, S. Nolte, A. Szameit, and P. Walther, Experimental boson sampling, Nat. Photonics 7, 540 (2013).

[7] A. Crespi, R. Osellame, R. Ramponi, D. J. Brod, E. F. Galvão, N. Spagnolo, C. Vitelli, E. Maiorino, P. Mataloni, and F. Sciarrino, Integrated multimode interferometers with arbitrary designs for photonic boson sampling, Nat. Photonics 7, 545 (2013).

[8] H. Wang, Y. He, Y.-H. Li, Z.-E. Su, B. Li, H.-L. Huang, X. Ding, M.-C. Chen, C. Liu, J. Qin, J.-P. Li, Y.-M. He, C. Schneider, M. Kamp, C.-Z. Peng, S. Höfling, C.-Y. Lu, and J.-W. Pan, High-efficiency multiphoton boson sampling, Nat. Photonics 11, 361 (2017).

[9] S. Paesani, Y. Ding, R. Santagati, L. Chakhmakhchyan, C. Vigliar, K. Rottwitt, L. K. Oxenløwe, J. Wang, M. G. Thompson, and A. Laing, Generation and sampling of quantum states of light in a silicon chip, Nat. Phys. 15, 925 (2019).

[10] H. Wang, J. Qin, X. Ding, M. C. Chen, S. Chen, X. You, Y. M. He, X. Jiang, L. You, Z. Wang, C. Schneider, J. J. Renema, S. Höfling, C. Y. Lu, and J. W. Pan, Boson Sampling with 20 Input Photons and a 60-Mode Interferometer in a $10^{14}$-Dimensional Hilbert Space, Phys. Rev. Lett. 123, 250503 (2019).

[11] H.-S. Zhong, et al., Quantum computational advantage using photons, Science 370, 1460 (2020).

[12] M. C. Tichy, M. Tiersch, F. De Melo, F. Mintert, and A. Buchleitner, Zero-Transmission Law for Multiport Beam Splitters, Phys. Rev. Lett. 104, 220405 (2010).
[13] F. Arute, K. Arya, R. Babbush, et al., Quantum supremacy using a programmable superconducting processor, Nature 574, 505 (2019).

[14] T. Giordani, F. Flamini, M. Pompili, N. Viggianello, N. Spagnolo, A. Crespi, R. Osellame, N. Wiebe, M. Walschaers, A. Buchleitner, and F. Sciarrino, Experimental statistical signature of many-body quantum interference, Nat. Photonics 12, 173 (2018).

[15] M. Walschaers, J. Kuipers, J.-D. Urbina, K. Mayer, M. Tichy, K. Richter, and A. Buchleitner, Statistical benchmark for BosonSampling, New J. Phys. 18, 032001 (2016).

[16] E. Pednault, J. Gunnels, G. Nannicini, L. Horesh, and R. Wisnieff, arXiv:1910.09534 [quant-ph] (2019).

[17] B.-G. Englert, Fringe Visibility and Which-Way Information: An Inequality, Phys. Rev. Lett. 77, 2154 (1996).

[18] C. Dittel, G. Dufour, G. Weihs, and A. Buchleitner, arXiv:1901.02810 [quant-ph] (2019).

[19] C. K. Hong, Z. Y. Ou, and L. Mandel, Measurement of Subpicosecond Time Intervals between Two Photons by Interference, Phys. Rev. Lett. 59, 2044 (1987).

[20] Y. L. Lim and A. Beige, Generalized Hong-Ou-Mandel experiments with bosons and fermions, New J. Phys. 7, 155 (2005)

[21] M. C. Tichy, M. Tiersch, F. Mintert, and A. Buchleitner, Many-particle interference beyond many-boson and manyfermion statistics, New J. Phys. 14, 093015 (2012).

[22] A. Perez-Leija, R. Keil, A. Kay, H. Moya-Cessa, S. Nolte, L.-C. Kwek, B. M. Rodríguez-Lara, A. Szameit, and D. N. Christodoulides, Coherent quantum transport in photonic lattices, Phys. Rev. A 87, 012309 (2013).

[23] A. Crespi, Suppression laws for multiparticle interference in Sylvester interferometers, Phys. Rev. A 91, 013811 (2015).

[24] C. Dittel, R. Keil, and G. Weihs, Many-body quantum interference on hypercubes, Quantum Sci. Technol. 2, 015003 (2017).

[25] N. Spagnolo, C. Vitelli, L. Aparo, P. Mataloni, F. Sciarrino, A. Crespi, R. Ramponi, and R. Osellame, Three-photon bosonic coalescence in an integrated tritter, Nat. Commun. 4, 1606 (2013).

[26] J. Carolan, C. Harrold, C. Sparrow, E. Martín-López, N. J. Russell, J. W. Silverstone, P. J. Shadbolt, N. Matsuda, M. Oguma, M. Itoh, G. D. Marshall, M. G. Thompson, J. C. Matthews, T. Hashimoto, J. L. O'Brien, and A. Laing, Universal linear optics, Science 349, 711 (2015).

[27] A. Crespi, R. Osellame, R. Ramponi, M. Bentivegna, F. Flamini, N. Spagnolo, N. Viggianiello, L. Innocenti, P. Mataloni, and F. Sciarrino, Suppression law of quantum states in a 3D photonic fast Fourier transform chip, Nat. Commun. 7, 10469 (2016).

[28] Z. E. Su, Y. Li, P. P. Rohde, H. L. Huang, X. L. Wang, L. Li, N. L. Liu, J. P. Dowling, C. Y. Lu, and J. W. Pan, Multiphoton Interference in Quantum Fourier Transform Circuits and Applications to Quantum Metrology, Phys. Rev. Lett. 119, 080502 (2017).

[29] S. Weimann, A. Perez-Leija, M. Lebugle, R. Keil, M. Tichy, M. Gräfe, R. Heilmann, S. Nolte, H. Moya-Cessa, G. Weihs, D. N. Christodoulides, and A. Szameit, Implementation of quantum and classical discrete fractional Fourier transforms, Nat. Commun. 7, 11027 (2016). 
[30] N. Viggianiello, F. Flamini, L. Innocenti, D. Cozzolino, M. Bentivegna, N. Spagnolo, A. Crespi, D. J. Brod, E. F. Galvao, R. Osellame, and F. Sciarrino, Experimental generalized quantum suppression law in Sylvester interferometers, New J. Phys. 20, 033017 (2018).

[31] S. Leedumrongwatthanakun, L. Innocenti, H. Defienne, T. Juffmann, A. Ferraro, M. Paternostro, and S. Gigan, Programmable linear quantum networks with a multimode fibre, Nat. Photonics 14, 139 (2020).

[32] M. Ehrhardt, R. Keil, L. J. Maczewsky, C. Dittel, M. Heinrich, and A. Szameit, Exploring complex graphs using three-dimensional quantum walks of correlated photons, Sci. Adv. 7, eabc5266 (2021).

[33] C. Dittel, G. Dufour, M. Walschaers, G. Weihs, A. Buchleitner, and R. Keil, Totally Destructive Many-Particle Interference, Phys. Rev. Lett. 120, 240404 (2018).

[34] C. Dittel, G. Dufour, M. Walschaers, G. Weihs, A. Buchleitner, and R. Keil, Totally destructive interference for permutation-symmetric many-particle states, Phys. Rev. A 97, 062116 (2018).

[35] M. C. Tichy, K. Mayer, A. Buchleitner, and K. Mølmer, Stringent and Efficient Assessment of Boson-Sampling Devices, Phys. Rev. Lett. 113, 020502 (2014).

[36] N. Viggianiello, F. Flamini, M. Bentivegna, N. Spagnolo, A. Crespi, D. J. Brod, E. F. Galvão, R. Osellame, and F. Sciarrino, Optimal photonic indistinguishability tests in multimode networks, Sci. Bull. 63, 1470 (2018).

[37] M. Walschaers, Signatures of many-particle interference, J. Phys. B At. Mol. Opt. Phys. 53, 43001 (2020).

[38] A. E. Jones, A. J. Menssen, H. M. Chrzanowski, T. A. W. Wolterink, V. S. Shchesnovich, and I. A. Walmsley, Multiparticle Interference of Pairwise Distinguishable Photons, Phys. Rev. Lett. 125, 123603 (2020).

[39] C. Dittel, Ph.D. thesis, University of Innsbruck (2019) https://diglib.uibk.ac.at/ulbtirolhs/content/titleinfo/ 3576984 .

[40] A. Perez-Leija, R. Keil, H. Moya-Cessa, A. Szameit, and D. N. Christodoulides, Perfect transfer of path-entangled photons in $J_{x}$ photonic lattices, Phys. Rev. A 87, 022303 (2013).

[41] Note that in Eq. (2), we attribute the columns and rows of $U^{J}$ to the input and output modes, respectively. In the literature, this is sometimes assumed to be the other way around.

[42] In other terms, the cycle structure underlying the permutation symmetry of a given input state defines the orders - two, three, . . N particles - of multiparticle interference contributions to the predicted event suppression.

[43] A. Szameit and S. Nolte, Discrete optics in femtosecondlaser-written photonic structures, J. Phys. B At. Mol. Opt. Phys. 43, 163001 (2010).

[44] T. Meany, M. Gräfe, R. Heilmann, A. Perez-Leija, S. Gross, M. J. Steel, M. J. Withford, and A. Szameit, Laser written circuits for quantum photonics, Laser Photonics Rev. 9, 363 (2015).

[45] Z. Y. Ou, J. K. Rhee, and L. J. Wang, Observation of Four-Photon Interference with a Beam Splitter by Pulsed Parametric Down-Conversion, Phys. Rev. Lett. 83, 959 (1999).
[46] W. P. Grice and I. A. Walmsley, Spectral information and distinguishability in type-II down-conversion with a broadband pump, Phys. Rev. A 56, 1627 (1997).

[47] Z. Y. Ou, J.-K. Rhee, and L. J. Wang, Photon bunching and multiphoton interference in parametric down-conversion, Phys. Rev. A 60, 593 (1999).

[48] H. D. Riedmatten, V. Scarani, I. Marcikic, A. Acín, W. Tittel, H. Zbinden, and N. Gisin, Two independent photon pairs versus four-photon entangled states in parametric down conversion, J. Mod. Opt. 51, 1637 (2004).

[49] Z. Y. Ou, Temporal distinguishability of an $N$-photon state and its characterization by quantum interference, Phys. Rev. A 74, 063808 (2006).

[50] V. S. Shchesnovich, Partial indistinguishability theory for multiphoton experiments in multiport devices, Phys. Rev. A 91, 013844 (2015).

[51] M. C. Tichy, Sampling of partially distinguishable bosons and the relation to the multidimensional permanent, Phys. Rev. A 91, 022316 (2015).

[52] G. J. Mendoza, R. Santagati, J. Munns, E. Hemsley, M. Piekarek, E. Martín-López, G. D. Marshall, D. Bonneau, M. G. Thompson, and J. L. O'Brien, Active temporal and spatial multiplexing of photons, Optica 3, 127 (2016).

[53] F. Lenzini, B. Haylock, J. C. Loredo, R. A. Abrahão, N. A. Zakaria, S. Kasture, I. Sagnes, A. Lemaitre, H. P. Phan, D. V. Dao, P. Senellart, M. P. Almeida, A. G. White, and M. Lobino, Active demultiplexing of single photons from a solid-state source, Laser Photonics Rev. 11, 1600297 (2017).

[54] T. Hummel, C. Ouellet-Plamondon, E. Ugur, I. Kulkova, T. Lund-Hansen, M. A. Broome, R. Uppu, and P. Lodahl, Efficient demultiplexed single-photon source with a quantum dot coupled to a nanophotonic waveguide, Appl. Phys. Lett. 115, 021102 (2019).

[55] L. Schweickert, K. D. Jöns, K. D. Zeuner, S. F. Covre Da Silva, H. Huang, T. Lettner, M. Reindl, J. Zichi, R. Trotta, A. Rastelli, and V. Zwiller, On-demand generation of background-free single photons from a solid-state source, Appl. Phys. Lett. 112, 093106 (2018).

[56] L. Hanschke, K. A. Fischer, S. Appel, D. Lukin, J. Wierzbowski, S. Sun, R. Trivedi, J. Vučković, J. J. Finley, and K. Müller, Quantum dot single-photon sources with ultra-low multi-photon probability, npj Quantum Inf. 4, 43 (2018).

[57] E. Schöll, L. Hanschke, L. Schweickert, K. D. Zeuner, M. Reindl, S. F. Covre da Silva, T. Lettner, R. Trotta, J. J. Finley, K. Müller, A. Rastelli, V. Zwiller, and K. D. Jöns, Resonance fluorescence of GaAs quantum dots with near-unity photon indistinguishability, Nano Lett. 19, 2404 (2019).

[58] M. C. Tichy, H.-T. Lim, Y.-S. Ra, F. Mintert, Y.-H. Kim, and A. Buchleitner, Four-photon indistinguishability transition, Phys. Rev. A 83, 062111 (2011).

[59] J. Carolan, J. D. A. Meinecke, P. J. Shadbolt, N. J. Russell, N. Ismail, K. Wörhoff, T. Rudolph, M. G. Thompson, J. L. O'Brien, J. C. F. Matthews, and A. Laing, On the experimental verification of quantum complexity in linear optics, Nat. Photonics 8, 621 (2014). 
[60] R. Heilmann, C. Greganti, M. Gräfe, S. Nolte, P. Walther, and A. Szameit, Tapering of femtosecond laser-written waveguides, Appl. Opt. 57, 377 (2018).

[61] T. Meany, M. Delanty, S. Gross, G. D. Marshall, M. J. Steel, and M. J. Withford, Non-classical interference in integrated 3D multiports, Opt. Express 20, 26895 (2012).

[62] A. Peruzzo, A. Laing, A. Politi, T. Rudolph, and J. L. O'Brien, Multimode quantum interference of photons in multiport integrated devices, Nat. Commun. 2, 1 (2011).

[63] A. Laing and J. L. O'Brien, arXiv:1208.2868 [quant-ph] (2012).

[64] S. Rahimi-Keshari, M. A. Broome, R. Fickler, A. Fedrizzi, T. C. Ralph, and A. G. White, Direct characteriza- tion of linear-optical networks, Opt. Express 21, 13450 (2013).

[65] M. Tillmann, C. Schmidt, and P. Walther, On unitary reconstruction of linear optical networks, J. Opt. (United Kingdom) 18, 114002 (2016).

[66] P. Kolenderski, W. Wasilewski, and K. Banaszek, Modeling and optimization of photon pair sources based on spontaneous parametric down-conversion, Phys. Rev. A 80, 013811 (2009).

[67] J. Zeuner, A. N. Sharma, M. Tillmann, R. Heilmann, M. Gräfe, A. Moqanaki, A. Szameit, and P. Walther, Integrated-optics heralded controlled-NOT gate for polarization-encoded qubits, npj Quantum Inf. 4, 13 (2018). 